\title{
Synthesis of new thiazole and thiazolyl derivatives of medicinal significant-a short review
}

\begin{abstract}
In the field of therapeutic science, thiazoles are of extraordinary importance, because of their potent and significant biological activities. Likewise thiazole and their derivatives are found in various powerful naturally and biologically active compounds which possess a broad spectrum of biological activity therefore, synthesis of this compound is of remarkable concern. This review primarily focuses on the updated research papers reported in literature for the synthesis of thiazole and thiazolyl compounds.
\end{abstract}

Keywords: thiazoles, thiazolyl, synthetic procedures, biological activity
Volume 2 Issue 2 - 2018

\author{
Weiam Hussein,' Gülhan Turan- \\ Zitouni $^{2}$ \\ 'Department of Pharmaceutical Chemistry,Aden University, \\ Yemen \\ ${ }^{2}$ Department of Pharmaceutical Chemistry, Anadolu University, \\ Turkey
}

Correspondence: Weiam Hussein, Anadolu University, Faculty of Pharmacy, Department of Pharmaceutical Chemistry, Eskișehir, Turkey, Tel +905074929053,

Email white.wf.rose@gmail.com

\section{Introduction}

The synthesis of heterocyclic rings has been a fascinating field in therapeutic science. Various heterocyclic compounds containing nitrogen and sulfur have flexible frameworks for drugs development and design. ${ }^{1}$ Thiazole is one of the most intensively studied classes of aromatic five-membered heterocyclics. It was first defined by Hantzsch and Weber in 1887. Thiazoles are important class of heterocyclic compounds, found in many powerful biologically active drugs such as Sulfathiazol (antimicrobial drug), Ritonavir (antiretroviral drug), Abafungin (antifungal drug) and Tiazofurin (antineoplastic drug). ${ }^{2}$ Thus, thiazole or thiazolyl moiety if it is present in any compound will show numerous biological activities such as antimicrobial \& antifungal, ${ }^{3}$ anti-inflammatory, ${ }^{4}$ anticancer, ${ }^{5}$ antihypertensive,${ }^{6}$ anti$\mathrm{HIV},{ }^{7}$ anticonvulsant ${ }^{8}$ and antidiabetic ${ }^{9}$ activities. Accordingly, this imperative ring framework keeps on pulling in consideration from the medicinal chemists and new synthetic way for the development of this heteroaromatic ring keeps on being developed. This short review mainly focuses on the research work reported in the recent literatures on various synthetic methods of thiazole and thiazolyl compounds.

\section{Synthesis of thiazole \& thiazolyl derivatives}

Santana et al. ${ }^{10}$ reported the synthesis of new substances belonging to thiosemicarbazones and thiazoles to develop powerful therapeutic agents for cancer treatment. The intermediate thiosemicarbazones were prepared in reaction between the 4'-trifluoromethylbenzaldehyde with the corresponding thiosemicarbazide under reflux and catalytic amount of $\mathrm{HCl}$. These intermediate compounds then react with different $\alpha$-halogenated ketones, to obtain the series with yields of $22 \%$ to $94 \%$ (Figure 1).

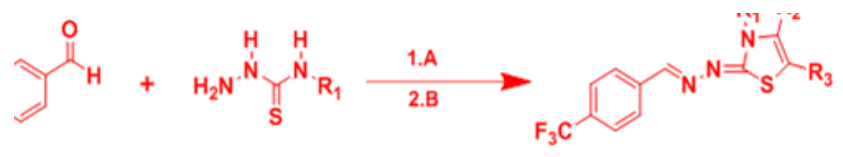

Figure I (A) Corresponding thiosemicarbazide, ethanol, $\mathrm{HCL}$, reflux,2-3h; (B) Corresponding haloketone, 2-propanol, rt, Ih.
Kaplancikli et al. ${ }^{11}$ reported a simple and three steps-reaction procedure for the synthesis of new benzimidazole-thiazole derivatives. 4-(1H-benzimidazol-1-yl)benzaldehyde was prepared by reacting 1H-benzimidazole and 4-fluorobenzaldehyde under microwave irradiation, then, the reaction between 4- $(1 \mathrm{H}-$ benzimidazol1-yl)benzaldehyde and hydrazine carbothioamide gave as a result 2-(4-(1H-benzimidazol-1-yl)benzylidene)hydrazine-1carbothioamide derivatives which on reaction with appropriate 2-bromoacetophenones produced the final compounds (Figure 2).

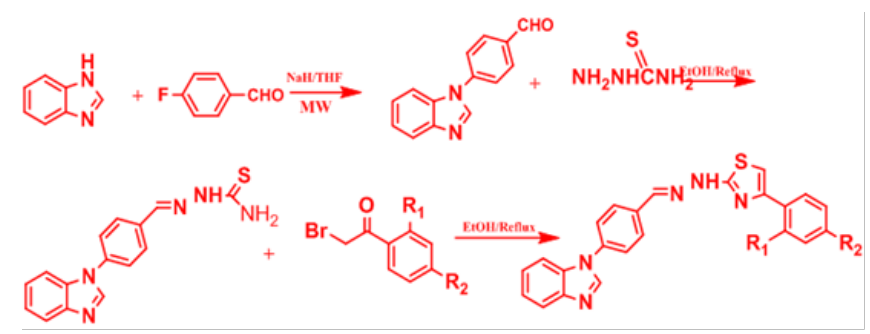

Figure 2 Synthesis of targeted compounds

Bikobo etal. ${ }^{12}$ synthesized a series of novel N-phenyl-4-(4-(thiazol2-yl)-phenyl)thiazol-2-amine and 3-(2-(phenylamino)thiazol-4yl)-benzamide ether derivatives First of all , 4-(a-bromoacetyl)benzonitrile was refluxed with $\mathrm{N}$-phenyl thiourea, to obtain 4-((2-phenylamino)thiazol-4-yl)-benzonitrile, which undergoes Willgerodt-Kindler reaction ( $\mathrm{FeS}$, TEA and $\mathrm{HCl}$ ) producing $\mathrm{H} 2 \mathrm{~S}$, to give of 4-((2-phenylamino)thiazol-4-yl)benzothioamide. This was then condensed with various halo-ketones in acetone or ethanol, to afford, N-phenyl-4-(4-(thiazol-2-yl)phenyl)thiazol-2-amine derivatives. In another case, N-phenylthiourea was heated with 2-hydroxy-5-(a-bromoacetyl) benzamide, and gave 2-hydroxy-5-(2(phenylamino)thiazol-4-yl)benzamide, which was then treated with $\mathrm{K}_{2} \mathrm{CO}_{3}$, in acetone-DMF and stirred at room temperature, to give the corresponding potassium phenolate. The later one was treated with a-halo-ketones, in order to give the 5-(2-(phenylamino)thiazol-4-yl) benzamide ethers in good yields (72-96\%) (Figure 3). 


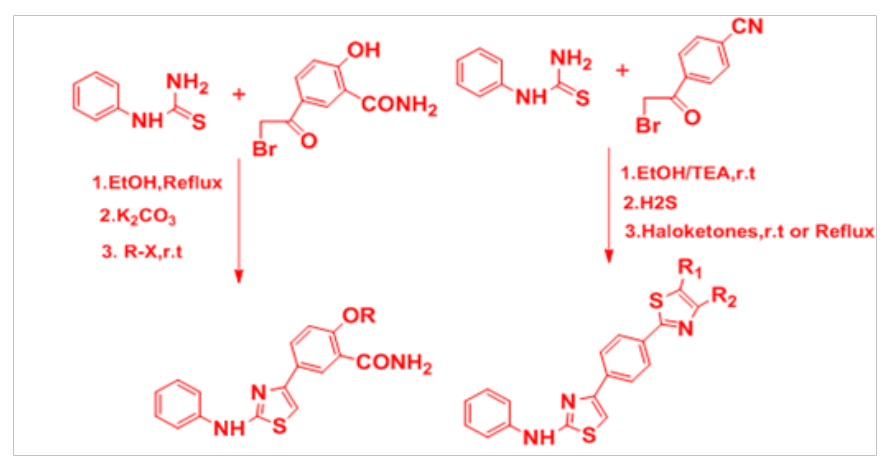

Figure 3 Synthesis procedure

Farghaly et al. $^{13}$ described the synthesis of new thiazole derivatives. The thiocroman-4-thiocarbohydrazone was used in this work as starting material for synthesis of new series of thiochromanes incorporating thiazole moiety by its reaction with a variety of hydrazonoyl chlorides using triethylamine as a catalyst (Figure 4).

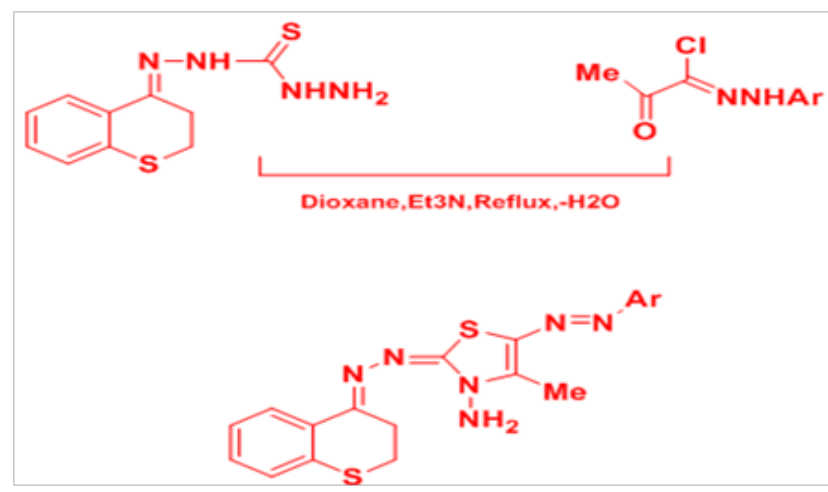

Figure 4 General way for synthesis

Turan-Zitouni et al. ${ }^{14}$ reported the synthesis of new 2-[2-[4-(ethyl/ phenyl)cyclohexylidene]hydrazinyl]-4-(4-substitutedphenyl) thiazole by two step synthesis, thus the reaction of 4-(ethyl/phenyl) cyclohexanone with thiosemicarbazide resulted to the formation of 2-[4-(ethyl/phenyl)cyclohexylidene]hydrazine-1-carbothioamide derivatives . Then equimolar quantities of above mentioned compounds with appropriate phenacyl bromide in ethanol as solvent resulted to the formation of the final compound (Figure 5).

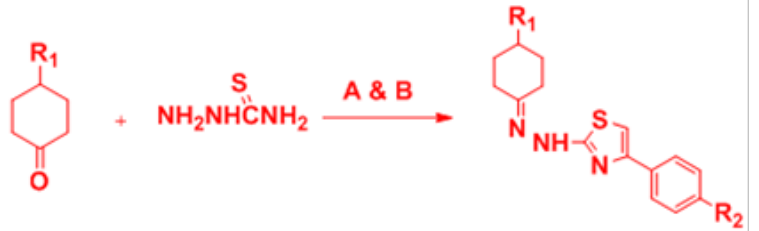

Figure 5 Synthesis of the compounds. Reactants and reagents: (A) EtOH, acetic acid, reflux, 2h, B. appropriate phenacyl bromide, EtOH, I-8 h, room temperature.

Tabbi et al. ${ }^{15}$ Synthesized new thiazolylpyrazoline derivatives by reacting substituted 3,5-diaryl-1-thiocarbamoyl-2-pyrazolines with phenacylbromides. The intermediate products were synthesized via the base catalyzed Claisen-Schmidt condensation. All the synthesized compounds displayed good antibacterial activity against K. pneumoniae and P. aeruginosa, with a MIC value of $200 \mu \mathrm{g} / \mathrm{mL}$ (Figure 6).

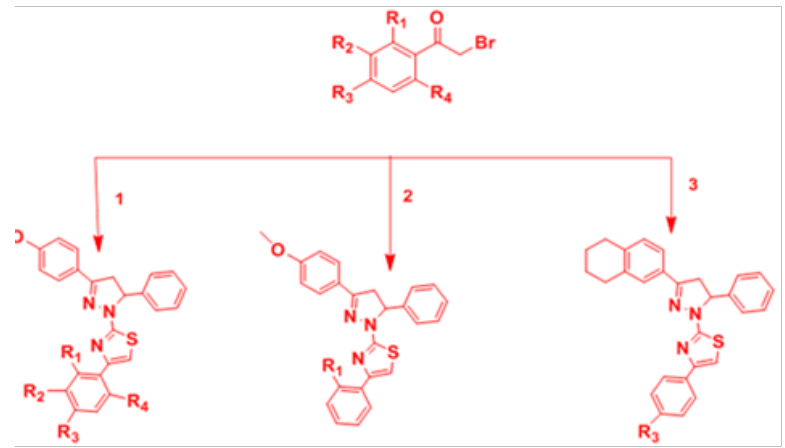

I.3-(4'-Methoxyphenyl)-5phenyl-2-pyrazolin- I-carbothioamide 2.3-(4'-methoxyphenyl)-5-phenyl- I-thiocarbomoyl-2-pyrazoline 3.5-phenyl-3-(5,6,7,8-tetrahydronaphthalen-2-yl)-I-thiocarbamoyl-2pyrazoline

Figure 6 Synthesis of title compounds.

Gomha et al. ${ }^{16}$ reported the synthesis of new thiazolyl-thiazole derivatives by the reaction of 5-acetyl-2-amino-4-methylthiazole with thiocarbohydrazide and thiosemicarbazide in absolute ethanol and in the presence of a catalytic amount of concentrated $\mathrm{HCl}$ as indicated in Figure 7.

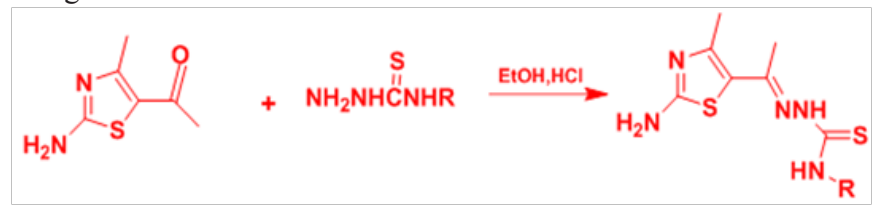

Figure 7 Synthesis of thiazole derivatives.

Yurttaş et al. ${ }^{17}$ described the synthesis of new 2-(4-arylpiperazine1-yl)-N-[4-(2-(4-substituted phenyl)thiazol-4-yl)phenyl]acetamide derivatives by a five-step reaction, thus different 4-substituted thiobenzamides $\left(\mathrm{H}, \mathrm{OCH}_{3}, \mathrm{Cl}, \mathrm{F}\right)$ were used as starting materials. They reacted with $\mathrm{N}$-[4-(2-bromoacetyl)phenyl]acetamide to obtain thiazole compounds. After deacetylation of these intermediates, 2-chloro-N-[4-(2-(4-substituted phenyl)-4-thiazolyl)phenyl] acetamides were obtained. Finally, 2-[4-(aryl)piperazin-1-yl]-N-[4-(2(4-substituted phenyl)thiazol-4-yl)phenyl]acetamide derivatives were afforded by the reaction of these intermediates and corresponding piperazine derivatives. All these compounds were evaluated for their antimicrobial and anticholinesterase activities (Figure 8).

Özkay et al. ${ }^{18}$ synthesized some hydrazone derivatives of thiazole to evaluate their anticholinesterase activities. Pyrrole-2-carboxaldehydes reacted with thiosemicarbazide in ethanol then, the thiosemicarbazones obtained were condensed with $\alpha$-bromoacetophenone derivatives (Hantzsch reaction) to give 1-substituted pyrrole-2-carboxaldehyde (4-(4-substituted phenyl)-1,3-thiazol-2-yl) hydrazones (Figure 9).

Penta et al. ${ }^{19}$ developed a novel one-pot synthesis of thiazoles and thiazolyl-pyrazole derivatives via multi component approach. The condensation of 3-(2-bromoacetyl)-4-hydroxy-6-methyl-2Hpyran-2-one, thiosemicarbazide and various carbonyl compounds gave corresponding thiazole and thiazolylpyrazole derivatives in good yields by using Hantzsch-Thiazole synthesis. In addition, this method does not include the use of organic solvents thus it is an 
environmentally friendly process (Figure 10).

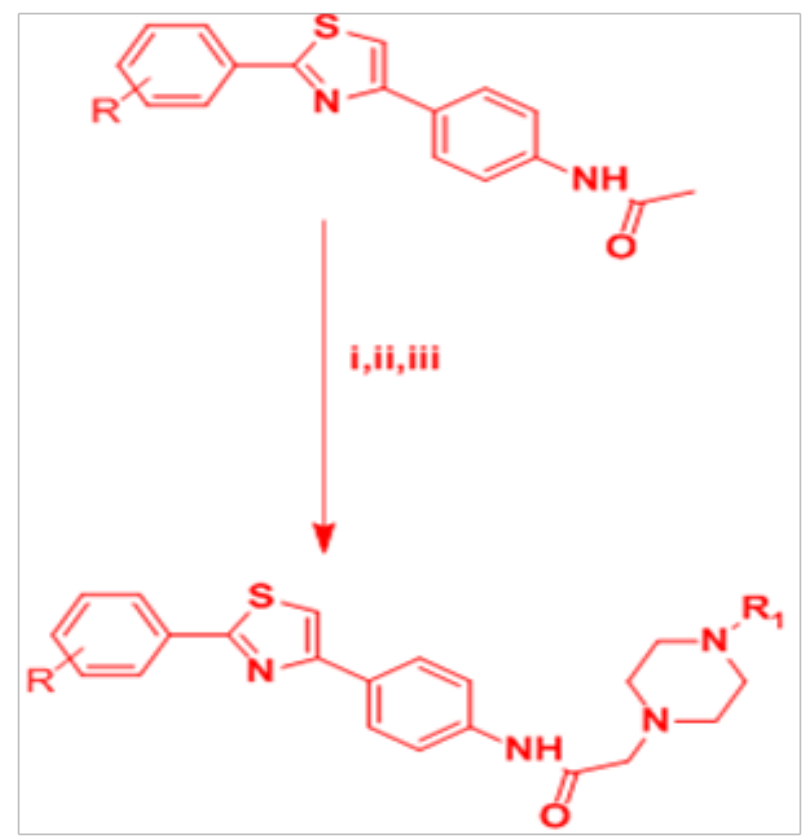

Figure 8 Synthesis of the compounds. Reagents are (i) $10 \% \mathrm{HCl}$, EtOH, reflux; (ii) chloroacetyl chloride, TEA, THF, r.t; and (iii) appropriate 4-substituted piperazine derivatives, $\mathrm{K} 2 \mathrm{CO} 3$, acetone, reflux.

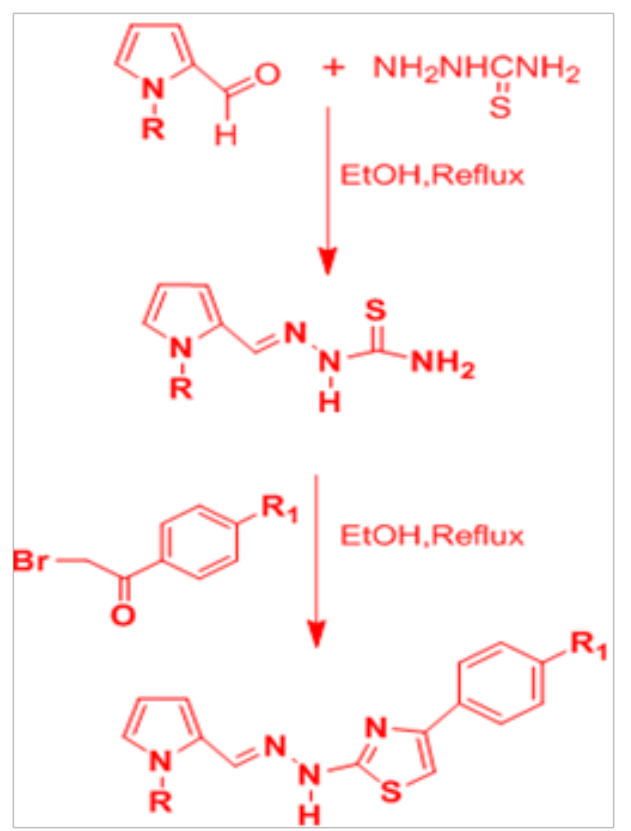

Figure 9 The synthetic protocol of the final compounds.

Kaplancikli et al. ${ }^{20}$ described the synthesis 2-[[(benzoxazole/ benzimidazole-2-yl)sulfanyl]acetylamino]thiazoles
Thus, in this study, 4-substituted-2-(chloroacetylamino) thiazole was prepared by reacting 2-amino-4-substituted-thiazole with chloroacetyl chloride. The reaction of 4-substituted-2-(chloroacetylamino)thiazole, benzimidazole/ benzoxazole-2-thiole , and anhydrous potassium carbonate in acetone gave the 4- substituted-2-[[(benzoxazole/ benzimidazole-2-yl)sulfanyl]acetylamino]thiazole derivatives as final products (Figure 11).

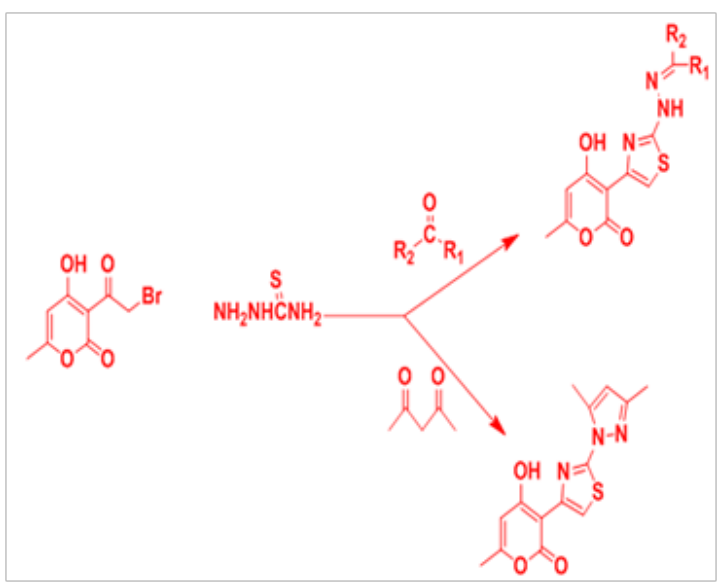

Figure 10 One-pot reaction synthesis.

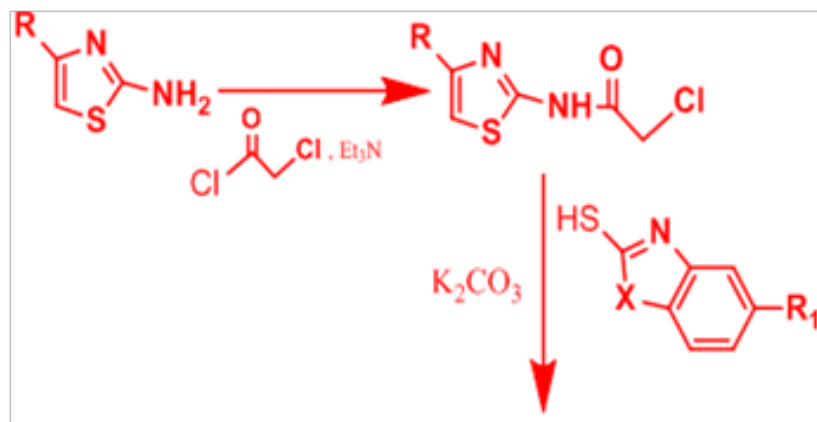

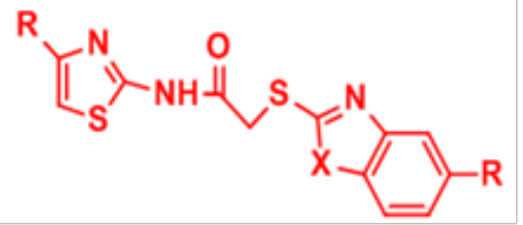

Figure II Synthesis of the compounds.

\section{Current development in the synthesis of thiazoles}

The synthetic significance of thiazole derivatives have been increased much by their recent medicinal applications as anticancer, antifungal, anti-inflammatory, antihypertensive, antiHIV, anticonvulsant and antidiabetic thus, thiazole chemistry has developed in steady state as we can see in many recent literatures. ${ }^{21,22}$ Based on this; many recent articles showed the use of palladium(II) acetate which catalyzes a really selective coupling of 4-substituted 2-aminothiazoles from vinyl azides and potassium thiocyanate, whereas iron(III) bromide supports the formation of 4-substituted 
5-thiocyano-2-aminothiazoles. ${ }^{23}$ In addition, a copper-catalyzed reaction of oxime acetates with isothiocyanates gives various 4-substituted and 4,5-disubstituted 2-aminothiazoles under mild reaction conditions via copper-catalyzed $\mathrm{N}-\mathrm{O}$ bond splitting, activation of vinyl sp2 $\mathrm{C}-\mathrm{H}$ bonds, and $\mathrm{C}-\mathrm{S} / \mathrm{C}-\mathrm{N}$ bond formations. ${ }^{24}$ Copper also, facilitated $[3+1+1]$-type condensation of oximes, anhydrides and potassiumthiocyanate $(\mathrm{KSCN})$ to give thiazoles in very good yields under very mild reaction conditions..$^{25,26}$ The uses of DABCO (1,4-diazabicyclo[2.2.2]octane) as an organocatalyst for synthesis of new thiazole derivatives in short reaction times and good to high yields was also reported. ${ }^{27}$ Due to reactivity of 2-amino-4,5,6,7tetrahydrobenzo[b]thiophene-3- carbonitrile towards thioglycolic acid the latter was used with different chemical reagents to give many thiazole derivatives. ${ }^{28}$

\section{Conclusion}

In the current years, numerous thiazole derivatives have been synthesized and subjected to fluctuate the biological activity of thiazole. In this article, we try to survey the simplest synthetic way for thiazole-based derivatives and feature their parts in new leads recognizable proof and medication disclosure. In this way the journey to investigate numerous more changes on thiazole moiety should be proceeded.

\section{Acknowledgements}

Authors contributed toward data analysis, drafting and revising the paper and agree to be accountable for all aspects of the work.

\section{Conflict of interest}

There is no conflict of interest.

\section{Reference}

1. Acheson RM. An introduction to the chemistry of heterocyclic compounds. $3^{\text {rd }}$ edn. India: Willy-Inter sciences; 2009.

2. Siddiqui N, Arshad MF, Ahsan W, et al. Thiazoles: A Valuable Insight into the Recent Advances and Biological Activities. Int J Pharm Sci Drug Res. 2009;1(3):136-143.

3. Vasu N, Goud BB, Kumari YB, et al. Design, Synthesis and Biological Evaluation of Some Novel Benzimidazole Based Thiazolyl Amines. Rasayan J Chem. 2013;6:201-206.

4. Singh N, Bhati SK, Kumar A. Thiazolyl/oxazolyl formazanyl indoles as potent anti-inflammatory agents. Eur $J$ Med Chem. 2008;43(11):2597-2609.

5. Luzina EL, Popov AV. Synthesis and anticancer activity of N-bis(trifluoromethyl)alkyl-N'-thiazolyl and N-bis(trifluoromethyl) alkyl-N'-benzothiazolyl ureas. Eur JMed Chem. 2009;44(12):4944-4953.

6. Turan-Zitouni G, Chevallet P, Kilic FS, et al. Synthesis of some thiazolylpyrazoline derivatives and preliminary investigation of their hypotensive activity. Eur J Med Chem. 2000;35(6):635-641.

7. Rawal RK, Tripathi R, Katti SB, et al. Design and synthesis of 2-(2,6-dibromophenyl)-3-heteroaryl-1,3-thiazolidin-4-ones as anti-HIV agents. Eur J Med Chem. 2008;43(12):2800-2806.

8. Satoh A, Nagatomi Y, Hirata Y, et al. Discovery and in vitro and in vivo profiles of 4-fluoro-N-[4-[6-(isopropylamino)pyrimidin-4-yl]1,3-thiazol-2-yl]-N-methylbenzamide as novel class of an orally active metabotropic glutamate receptor 1 (mGluR1) antagonist. Bioorg Med Chem Lett. 2009;19(18):5464-5468.

9. Iino T, Tsukahara D, Kamata K, et al. Discovery of potent and orally active 3-alkoxy-5-phenoxy-N-thiazolyl benzamides as novel allosteric glucokinase activators. Bioorg Med Chem. 2009;17(7):2733-2743.

10. de Santana TI, Barbosa MO, Gomes PATM, et al. Synthesis, anticancer activity and mechanism of action of new thiazole derivatives. Eur J Med Chem. 2018;144:874-886.

11. Kaplancikli ZA, Levent S, Osmaniye D, et al. Synthesis and Anticandidal Activity Evaluation of New Benzimidazole-Thiazole Derivatives. Molecules. 2017;22(12):E2051.

12. Bikobo DSN, Dan C Vodnar, Stana A, et al. Synthesis of 2-phenylaminothiazole derivatives as antimicrobial agents. Journal of Saudi Chemical Society. 2017;21(7):861-868.

13. Farghaly TA, Abdallah MA, Khedr MA, et al. Synthesis, Antimicrobial Activity and Molecular Docking Study of Thiazole Derivatives. $J$ Heterocyclic Chem. 2017;54(4):2417-2425.

14. Turan-Zitouni G, Çavuşoğlu BK, Sağlık BN, et al. Synthesis and antimicrobial activities of some novel thiazole compounds. Turk $J$ Biochem. 2017;1-8.

15. Tabbi A, Kaplancikli ZA, Tebbani D, et al. Synthesis of novel thiazolylpyrazoline derivatives and evaluation of their antimicrobial activities and cytotoxicities. Turk J Chem. 2016;40:641-654.

16. Gomha SM, Salaheldin TA, Hassaneen HME, et al. Synthesis, Characterization and Molecular Docking of Novel Bioactive ThiazolylThiazole Derivatives as Promising Cytotoxic Antitumor Drug. Molecules. 2016;21(1):3.

17. Yurttaş 1, Özkay Y, Gençer HK, et al. Synthesis of Some New Thiazole Derivatives and Their Biological Activity Evaluation. Journal of Chemistry. 2015;2015:1-7.

18. Özkay Y, Yurttaș L, Mohsen UA, et al. Study on ThiazolylHydrazone Derivatives as Acetylcholinesterase Inhibitors. MÜSBED. 2014;4(1):38-42.

19. Penta S, Vedula RR. A facile one-pot synthesis of thiazoles and thiazolylpyrazole derivatives via multicomponent approach. Org Commun. 2012;5(3):143-149.

20. Kaplancikli ZA, Turan-Zitouni G, Revial G, et al. Synthesis and study of antibacterial and antifungal activities of novel 2-[[(benzoxazole/ benzimidazole-2-yl)sulfanyl] acetylamino]thiazoles. Arch Pharm Res. 2004;27(11):1081-1085.

21. Chhabria TM, Patel S, Modi P, et al. Thiazole: A Review on Chemistry, Synthesis and Therapeutic Importance of its Derivatives. Curr Top Med Chem. 2016;16(26):2841-2862.

22. Chhabria MT, Patel S, Modi P, et al. Thiazole: A Review on Chemistry, Synthesis and Therapeutic Importance of its Derivatives. Curr Top Med Chem. 2016;16(26):2841-2862.

23. Chen B, Guo S, Guo X, et al. Selective Access to 4-Substituted 2-Aminothiazoles and 4-Substituted 5-Thiocyano-2-aminothiazoles from Vinyl Azides and Potassium Thiocyanate Switched by Palladium and Iron Catalysts. Org Lett. 2015;17(19):4698-4701.

24. Tang X, Zhu Z, Qi C, et al. Copper-Catalyzed Coupling of Oxime Acetates with Isothiocyanates: A Strategy for 2-Aminothiazoles. Org Lett. 2016;18(2):180-183.

25. Tang X, Yang J, Zhu Z, et al. Access to Thiazole via Copper-Catalyzed $[3+1+1]$-Type Condensation Reaction under Redox-Neutral Conditions. J Org Chem. 2016;81(22):11461-11466.

26. Gomha SM, Abdelaziz MR, Abdel-Aziz HM, et al. Green Synthesis and Molecular Docking of Thiazolyl-thiazole Derivatives as Potential Cytotoxic Agents. Mini Rev Med Chem. 2017;17(9):805-815.

27. Mohareb RM, Abdallah AEM, Ahmed EA. Synthesis and cytotoxicity evaluation of thiazole derivatives obtained from 2-amino4,5,6,7-tetrahydrobenzo[b]thiophene- 3-carbonitrile.Acta Pharm. 2017;67(4):495-510. 